\title{
eJRIEPS
}

Ejournal de la recherche sur l'intervention en éducation physique et sport

Numéro spécial 3 | 2020

Actes de la 11 ème Biennale de l'ARIS, Lille, 19-21 juin

2018

\section{Le « vouloir-faire débutant » de stagiaires en EPS : instruire et éduquer}

\section{Élisabeth Magendie}

\section{OpenEdition}

Journals

Édition électronique

URL : http://journals.openedition.org/ejrieps/4437

ISSN : 2105-0821

Éditeur

ELLIADD

\section{Référence électronique}

Élisabeth Magendie, "Le « vouloir-faire débutant » de stagiaires en EPS : instruire et éduquer », eJRIEPS [En ligne], Numéro spécial 3 | 2020, mis en ligne le 01 janvier 2020, consulté le 18 février 2020. URL : http://journals.openedition.org/ejrieps/4437

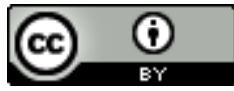

La revue eJRIEPS est mise à disposition selon les termes de la Creative Commons Attribution 4.0 International License. 


\section{Le « vouloir-faire débutant » de stagiaires en EPS : instruire et éduquer}

Élisabeth Magendie*

*Laboratoire Cultures, éducation, Société (LACES), EA4140, Université de Bordeaux, France.

Résumé

Cette recherche vise à identifier le "vouloir-faire débutant » qui engage des stagiaires EPS en deuxième année de master, c'est-à-dire ce dont ils se sentent responsables et pour lequel ils ont une obligation morale. Elle s'efforce de comprendre comment, dans un contexte d'intervention en mutation, ces derniers construisent ou peinent à construire de nouvelles responsabilités. Nous appréhendons pour ce faire, dans une perspective de didactique professionnelle, les éléments contextuels renvoyant aux différents horizons institutionnel, culturel et social, qui organisent leur activité lorsqu'ils interviennent auprès des élèves. Nous avons identifié deux grandes responsabilités qui sont engagées, souhaitées ou reportées par les stagiaires observés. La première renvoie à un impératif d'instruction ; la seconde à un impératif d'éducation. Après avoir discuté les résultats, nous présentons quelques implications pour la formation en guise de conclusion.

Mots clés: responsabilité, vouloir-faire, prescription, stagiaire, EPS, didactique professionnelle

\section{Summary}

This research aims to identify the "beginner willingness" that engages PE trainees entering the profession, i.e. what they feel responsible for and for which they have a moral obligation. It strives to understand how, in a context of changing intervention, they construct or struggle to construct new responsibilities. To do this, we apprehend, from a professional didactic perspective, the contextual elements referring to the different institutional, cultural and social horizons that organize the trainees' activities We have identified two main responsibilities 


\section{eJRIEPS spécial 3 (janvier 2020)}

that are engaged, desired or postponed by trainees. The first refers to an educational imperative; the second to an educational imperative. After discussing the results, we present some implications for training as a conclusion.

Key words : responsibility, willingness, prescription, trainee, PE, professional didactic

\section{Introduction}

L'activité physique, quelle que soit sa forme, est souvent utilisée, justifiée et légitimée au regard de valeurs vertueuses dont elle serait intrinsèquement porteuse. Elle favoriserait la santé, le bien être psychologique, l'intégration sociale, le mieux vivre ensemble ou la réduction des inégalités. Ainsi, peut-on lire par exemple dans le rapport de Deguilhem et Juanico $(2016$, p. 8) :

«Pour les enfants et adolescents, le sport, vecteur de respect des autres et des règles, joue un rôle éducatif. À l'école, autant qu'en dehors, les activités physiques et sportives peuvent être un lieu d'épanouissement des élèves, permettant de développer leur confiance en eux et de s'impliquer volontairement dans une activité. [...] Par son impact sur la concentration, la confiance en soi et la motivation à l'école, le sport est un facteur de réussite scolaire. ”

Ces vertus sont également évoquées dans les programmes d'EPS : "l'EPS aide tous les collégiens et collégiennes à acquérir de nouveaux repères sur soi, sur les autres, sur l'environnement, pour construire une image positive de soi dans le respect des différences. " (MENESR, 2015a, p. 295). Ainsi est-il attendu par exemple des enseignants d'EPS qu'ils apprennent à leurs élèves de cycle 4 : à accepter la défaite et gagner avec modestie et simplicité ; à prendre et assumer des responsabilités au sein d'un collectif pour réaliser un projet ou remplir un contrat ; à agir avec et pour les autres, en prenant en compte les différences ; ou bien encore à entretenir leur santé (MENESR, 2015a, p. 296).

Ces prescriptions qui organisent en partie le travail des enseignants (Saujat \& Serres, 2015) s'intègrent dans un système plus large qui tend de plus en plus à se rapprocher d'une prescription infinie (Daniellou, 2002) : des finalités de l'école qui se brouillent (transmettre des connaissances, socialiser, transmettre les valeurs de la république, permettre l'épanouissement des élèves, favoriser l'insertion professionnelle, ....) et une diversification des missions, fonctions et tâches assignées aux enseignants (Saujat \& Serres, 2015). Cette 


\section{eJRIEPS spécial 3 (janvier 2020)}

prescription infinie d'objectifs va de pair avec une contradictoire sous-prescription chronique des moyens pour les atteindre (Saujat, 2010). D'où des souffrances issues des doutes sur les finalités et le contenu même du travail, et un sentiment d'impuissance à agir (Rayou, 2014). Ce malaise des enseignants s'accroît du fait de l'exigence croissante d'imputabilité de leurs actes, liée au pilotage par les résultats du système scolaire.

Néanmoins, la responsabilité des enseignants peut aussi s'appréhender, non pas comme « réponse de ses actes ", mais comme "réponse à un donné » (Jonas, 1979). De fait, les difficultés rencontrées par les enseignants tiennent également à des formes de surprescription (Rayou, 2014) qui les obligent ou engagent leur responsabilité, et qui ont des conséquences objectives sur leurs démarches d'intervention. Les sur-prescriptions se situent en premier lieu sur un axe vertical sur lequel se trouvent : les prescriptions « descendantes " primaires (programmes, instructions et recommandations pédagogiques, évaluation, etc.) et secondaires (recommandations des formateurs) ; et les prescriptions « ascendantes " (Saujat, 2010), en particulier les demandes venues des élèves ou de leurs parents mais aussi celles qui « remontent » des collectifs de travail (Saujat \& Serres, 2015). Les sur-prescriptions se situent en second lieu sur un axe horizontal qui relie les enseignants entre eux (Rayou, 2014). Elles ont ici la forme d'auto-prescriptions correspondant selon le cas à des vulgates pédagogiques, des doxas, ou des succédanés de préconisations (Rayou, 2014).

Ces auto-prescriptions sont particulièrement prégnantes chez les stagiaires, qui ont tendance à adhérer aux conceptions et pratiques de leurs aînés ou de leurs pairs plutôt qu'à suivre les préconisations du centre de formation (Rayou, 2008). Face au choc de la réalité, ils prennent rapidement conscience de la complexité du métier et renoncent souvent à leurs idéaux (Roux-Perez, 2012). À la recherche de solutions fonctionnelles, voire de stratégies de survie, ils redéfinissent les tâches prescrites en distinguant une prescription maximale et exigeante et une prescription minimale, moins exigeante et réaliste (Daguzon, 2009). Ils recourent alors à des pratiques qui peuvent être jugées régressives par l'orthodoxie didactique ou pédagogique (Goigoux, Ria \& Toczek-Capelle, 2009).

Ferone (2010) a pu montrer que la pression des prescriptions ascendantes était accentuée par les réseaux horizontaux sur internet qui ont pour conséquence de faciliter la circulation des vulgates et des doxas chez les stagiaires en formation notamment. L'auteur montre, à partir d'une analyse des échanges sur des blogs de formation, que les stagiaires se soucient de mettre en activité les élèves en recourant à des activités ludiques, mais n'évoquent pratiquement jamais les apprentissages des élèves. De même, selon Butlen, Charles- 


\section{eJRIEPS spécial 3 (janvier 2020)}

Pezard, et Masselot (2009), les débutants se heurtent très vite aux tensions entre les logiques d'apprentissage et les logiques de socialisation et vivent par conséquent en concurrence leur mission d'instruction, liée à la transmission des savoirs, et leur mission d'éducation, liée au développement du bien-être et du devoir-être (Leleux, 2014). Adhérant à la doxa selon laquelle un travail de socialisation est un préalable aux apprentissages, ils s'efforcent avant tout de faire accepter les règles de fonctionnement aux élèves et de les enrôler rapidement dans les tâches scolaires pour obtenir le calme. Cherchant par ailleurs à faire réussir les élèves, leur donner confiance et les rassurer, plutôt qu'à les faire apprendre, ils ont tendance à simplifier les tâches qu'ils proposent.

Cet usage débutant du genre professionnel enseignant, c'est-à-dire des systèmes ouverts de façons de faire et de penser partagées par les enseignants (Saujat, 2004), se caractérise par des configurations d'activité provisoirement stabilisées, dans lesquelles se reconnaissent les enseignants débutants (Saujat \& Serres, 2015). II témoigne des préoccupations partagées par les débutants et manifeste leur vouloir-faire (Saujat \& Serres, 2015). C'est cette question du « vouloir-faire débutant » qui nous intéresse dans le cadre de cette étude. Nous voulons identifier le « vouloir-faire débutant » qui engage les stagiaires en formation, c'est-à-dire ce dont ils se sentent responsables et pour lequel ils ont une obligation morale au sens défini par Jonas (1979, p. 132) :

« II y a encore un tout autre concept de responsabilité qui ne concerne pas le calcul ex post facto de ce qui a été fait, mais la détermination de ce qui est à faire ; un concept en vertu duquel je me sens responsable non en premier lieu de mon comportement et de ses conséquences, mais de la chose qui revendique mon agir. 》

Pour accéder au «vouloir-faire débutant » et ainsi rendre compte des responsabilités engagées ou souhaitées par des enseignants stagiaires, nous cherchons à identifier les éléments contextuels qui organisent leurs actions. En agissant de la sorte, nous serons mieux à même de comprendre comment, dans un contexte d'intervention en mutation, ils construisent ou peinent à construire de nouvelles responsabilités.

\section{Orientations théoriques et méthodologiques}

\subsection{La conceptualisation dans l'activité professionnelle}

Pour accéder aux éléments contextuels qui organisent l'activité des stagiaires, nous partons de l'analyse de situations singulières en nous référant à l'approche de la didactique professionnelle (Pastré, 2011). Cette approche poursuit la visée du développement des 


\section{eJRIEPS spécial 3 (janvier 2020)}

compétences. Sa particularité est de coupler les méthodes d'analyse du travail issues de l'ergonomie francophone (Leplat, 1997) avec la théorie de la " conceptualisation dans l'action » d'inspiration piagétienne (Pastré, Mayen, \& Vergnaud, 2006).

Afin de repérer comment se fait la conceptualisation dans l'activité professionnelle, l'analyse s'attache à remonter jusqu'à son organisation, c'est-à-dire aux schèmes qui sont mobilisés dans différentes classes de situations (Vergnaud, 1996). Vergnaud (1996, p. 283) définit le schème comme " une organisation invariante de la conduite dans une classe de situations données » analysable en différentes composantes : un but, des sous-buts ; des possibilités d'inférence à partir de prises d'informations sur les situations, prises en charge par des règles d'action ou raisonnements conditionnels ; des invariants opératoires (concepts en actes et théorèmes en actes ou principes tenus pour vrais).

Les composantes du schème n'étant pas directement accessibles, il est nécessaire de recourir à l'observation, la verbalisation et l'analyse pour les faire émerger (Tourmen, 2014). Selon Mayen, Ferron, Humblot, et Bazil, (2006, p. 74) « c'est la confrontation à la situation qui permet au professionnel de verbaliser ce qu'il fait, dans quel but il le fait, avec quelles anticipations, à la suite de quelles prises d'informations. II est difficile, hors de l'action et sans une méthode, d'aboutir à un tel résultat. »

Aussi, pour étudier les invariants de l'organisation de l'action des stagiaires, nous organisons des entretiens d'auto-confrontation simple puis des entretiens d'autoconfrontation croisée conduit collectivement en nous inspirant des propositions de Goigoux (2007).

Si les entretiens d'auto-confrontation simple sont souvent utilisés par les chercheurs en didactique professionnelle (Pastré, 2011), c'est parce qu'ils permettent de susciter une parole sur l'activité de travail non déconnectée des traces de son déroulement (Tourmen, 2014). Le recours aux entretiens d'auto-confrontation croisée conduit collectivement, qui s'inspire des méthodes préconisées en clinique de l'activité (Clot, Prot, \& Werthe, 2001), est en revanche moins fréquent. Utilisé à d'autres fins que le développement du pouvoir d'agir (Clot et al., 2001), il a pour intérêt d'offrir aux stagiaires la possibilité d'une conceptualisation croissante de leur activité et d'approcher dans le même temps les principes qui assurent la cohérence interne de leur activité (Goigoux, 2007). Il est nécessaire pour cela de recourir à une méthodologie d'analyse précise (Pastré, 2011). Ainsi, pour identifier les principes tenus pour vrais notamment, qui, comme toute connaissance " en acte ", n'ont pas besoin d'être énoncés pour être effectifs, deux cas sont possibles : soit le principe tenu pour vrai est directement formulé par le sujet, il suffit alors de s'assurer qu'il permet bien de couvrir 


\section{eJRIEPS spécial 3 (janvier 2020)}

l'ensemble des autres énoncés ; soit le principe tenu pour vrai n'a pas été explicité, il est alors nécessaire que le chercheur formule lui-même le principe général qui fonde l'ensemble des autres énoncés. Selon Pastré (2011, p. 192), « c'est le pouvoir inférentiel attribué à ce jugement (ou principe) reconstruit qui est lindice d'un choix pertinent ».

\section{2. Éléments contextuels et responsabilités}

Après avoir analysé l'activité enseignante, de façon à remonter jusqu'à son organisation, et donc aux composantes des schèmes mobilisés en situation, nous procédons à un deuxième temps d'analyse qui consiste cette fois-ci à remonter des conceptualisations jusqu'aux éléments contextuels susceptibles d'avoir participé à leur production.

Les éléments contextuels ne sont pas donc pas posés a priori (comme facteur prédéterminant) mais a posteriori, en partant de l'analyse de l'activité in situ. Ils apparaissent lors de l'analyse de ce que dit le professionnel, seul ou avec d'autres, sur son activité. Cela signifie que l'analyse que nous menons, qui cherche à rendre compte de l'organisation de l'activité de l'enseignant, permet également de comprendre son rapport aux élèves, à l'établissement, aux prescriptions mais aussi au métier (Saussez, 2014), et ainsi d'identifier les éléments contextuels qui orientent et guident son activité. C'est à partir de cette analyse que nous mettons ensuite en évidence les responsabilités engagées, reportées ou souhaitées par les enseignants.

\section{Problématique et questions de recherche}

Dans le cadre de cette recherche, nous cherchons ainsi à identifier le « vouloir-faire débutant " qui engage des stagiaires en EPS entrant dans le métier afin de comprendre comment, dans un contexte d'intervention en mutation, ces derniers construisent ou peinent à construire de nouvelles responsabilités. Nous appréhendons pour ce faire, les éléments contextuels renvoyant aux différents horizons institutionnel, culturel et social, qui orientent et guident leur activité dans telle ou telle direction.

Nous tentons plus précisément de répondre à la question de recherche suivante : quelles sont les responsabilités engagées, souhaitées ou reportées par les stagiaires qui résultent de ces effets de contexte? 


\section{eJRIEPS spécial 3 (janvier 2020)}

\section{Méthodologie de recueil et de traitement des données}

\subsection{Recueil des données}

Les données sur lesquelles nous nous appuyons pour mener à bien ce travail proviennent de recherches antérieures dont l'objectif était de savoir comment se fait la conceptualisation dans l'activité professionnelle de stagiaires EPS (Magendie, 2014, 2016). II s'agissait, en référence aux préconisations de Pastré (2011), de focaliser l'analyse sur la recherche des invariants opératoires au cœur des schèmes, et plus précisément sur les principes tenus pour vrais qui renvoient aux différentes stratégies mobilisées par les stagiaires en rapport à une même classe de situations.

Ces recherches ont été conduites à partir de l'analyse de l'activité de 5 stagiaires (4 garçons : Théo, José et Tom en collège et Yan en lycée, et 1 fille : Marie en collège) en deuxième année de master MEEPS (métiers de l'enseignement en éducation physique et sportive) au cours de l'année 2012-2013.

Chaque stagiaire a été filmé et enregistré lors d'une séance d'apprentissage qui a eu lieu vers la fin du premier trimestre. Nous avons réalisé avec chacun d'entre eux un entretien d'auto-confrontation simple (EAS) en suivant les recommandations de Goigoux (2007). Lors de l'entretien les stagiaires étaient invités à commenter leur activité. Ils devaient centrer leurs commentaires sur les éléments invariants de leur activité. L'idée était qu'ils expliquent en quoi le déroulement des actions observées était habituel et dans quelle mesure il était similaire à celui qu'ils réalisent dans des situations comparables.

Au cours de l'entretien, nous avons eu recours à plusieurs types de questions. Pour identifier les buts de l'activité, souvent multiples et contradictoires (Leplat, 1997), nous avons posé des questions comme : «Quel but privilégies-tu dans ce cas-là ? Qu'est-ce qui risque de se passer si tu ne fais pas de cette façon ici et maintenant ? Quelles sont les conséquences de tes choix ? etc. » (Mayen et al., 2006). Pour identifier les principes tenus pour vrais, nous avons posé des questions qui invitaient à la généralisation (Mayen et al., 2006) : « Est-ce que tu fais souvent comment cela ? Est-ce que c'est une façon d'habituelle d'agir pour toi ? Comment les élèves se comportent-ils habituellement, ou se comportent-ils tous de la même manière ? »

Nous avons aussi réalisé un entretien d'auto-confrontation croisée (EAC) conduit collectivement avec les quatre garçons. II a eu lieu à la fin du premier trimestre également. Les stagiaires devaient à tour de rôle comparer leurs modes opératoires : «Tu fais comme cela toi ? »(Yvon \& Garon, 2006). Les échanges devaient porter sur ce qui faisait écho, par similitude ou par contraste à ses propres manières de faire, à ses interrogations, à ses 


\section{eJRIEPS spécial 3 (janvier 2020)}

convictions ou à ses valeurs (Goigoux, 2007). Tout au long du visionnage de la séance, les stagiaires pouvaient s'exprimer et débattre. Un tel cadre dialogique donnait à chacun l'occasion de revenir sur ses propres expériences et de formuler les buts et les principes tenus pour vrais qui orientent et guident ses actions.

\subsection{Accès aux composantes du schème}

Afin de pouvoir répondre à notre question de recherche, nous avons traité ces données en deux étapes. Lors de la première, nous avons cherché à éclairer les composantes du schème en répondant aux questions d'analyse suivantes :

- $\quad$ De quelle situation s'agit-il ?

- $\quad$ Quels sont le(s) but(s) visé(s) ?

- Quels sont les principes tenus pour vrais ?

Nous avons présenté les données d'observation et d'EAS pour chacun des stagiaires dans un tableau à quatre volets rendant compte respectivement : 1) de la succession temporelle des actions, 2) de la description des actions des enseignants et des élèves, 3) des communications en cours de séance et 4) du verbatim de l'entretien. Nous avons ensuite codé les passages de l'entretien au cours desquels des buts ou des principes tenus pour vrais étaient évoqués ou bien à partir desquels il était possible d'inférer ces éléments. La reconnaissance des principes tenus pour vrais, lorsqu'ils étaient exprimés, s'est faite à partir de leur forme : ce sont de propositions assertives qui interviennent le plus souvent non pas en réponse à une question mais plutôt au détour du récit sur l'activité de travail (Tourmen, 2014).

Nous avons entrepris ensuite un travail de comparaison intra et interindividuelle. Pour chaque classe de situations, nous avons relevé les buts et les principes tenus pour vrais " génériques " et " spécifiques ». Pour clore cette première étape d'analyse nous avons analysé les données de l'EAC conduit collectivement afin de préciser, d'affiner et réorganiser éventuellement les premiers résultats.

\subsection{Des éléments contextuels aux responsabilités}

Lors de la deuxième étape, notre démarche d'analyse, s'est apparentée en premier lieu à une analyse thématique (Paillé \& Mucchielli, 2003). Nous avons réalisé l'analyse en nous appuyant, non pas sur l'ensemble du corpus (puisque celui-ci avait déjà été analysé), mais sur les extraits de corpus auxquels étaient associés les buts et les principes tenus vrais identifiés. Nous sommes ainsi remontés des buts et des principes tenus pour vrais aux 


\section{eJRIEPS spécial 3 (janvier 2020)}

éléments contextuels qui permettent parfois de les expliquer (ce n'est pas toujours le cas). Nous avons repéré les éléments contextuels à partir des formulations justificatives énoncées par les stagiaires telles que : "Je fais cela... parce que... "; " J'ai l'habitude de... parce que... " ; "On m'a dit de faire comme ceci... "; "C'est écrit dans les programmes... »; « Tout le monde fait comme cela... », "C'est important de... », etc. Nous n'avions par conséquent pas de grille thématique a priori. Nous disposions néanmoins d'un plan général de codage indiquant les grandes catégories de prescriptions à partir desquelles réaliser l'analyse thématique inductive (Miles \& Huberman, 2003): les prescriptions descendantes (primaire et secondaire) ; les prescriptions ascendantes ; les prescriptions horizontales (doxa, succédanés et vulgates). Ce travail s'est poursuivi, selon la même logique, par l'analyse des EAC.

L'analyse a consisté en second lieu à mettre en regard les composantes du schème et les éléments contextuels afin de voir quelles étaient les responsabilités : engagées (ce qu'ils font qu'ils considèrent devoir faire) ; souhaitées (ce qu'ils ne font pas qu'ils considèrent devoir faire mais qu'ils aimeraient faire) ; ou reportées par les stagiaires (ce qu'ils ne font pas qu'ils considèrent devoir faire mais qu'ils disent vouloir faire plus tard). Nous avons relevé les éléments de discours renvoyant à la détermination de ce qui est à faire selon les stagiaires en tirant parti de termes tels que : en conséquence, donc, à cause de, etc. (Miles \& Huberman, 2003). La formulation des responsabilités s'est faite selon une démarche similaire à celle que nous avons utilisée pour formuler les principes tenus pour vrais. La différence réside dans le degré d'inférence et la distance prise par rapport aux propos tenus. Le tableau ci-dessous illustre la façon dont nous avons procédé. 
Tableau I : méthode d'identification des éléments contextuels et des responsabilités engagées, souhaitées ou reportées

\section{Extrait EAS}

\begin{tabular}{|c|c|c|}
\hline \multicolumn{3}{|c|}{ Extrait EAS } \\
\hline \multicolumn{3}{|c|}{$\begin{array}{l}\text { J'ai découpé la compétence attendue, je me la suis bien relue, les fiches ressources } \\
\text { surtout. Je me base sur ça au départ, parce que sinon on ne sait pas sur quoi partir. } \\
\text { [...] Je découpe, je vois globalement les différents éléments et je me dis va falloir } \\
\text { bosser tout ça. }\end{array}$} \\
\hline Buts visés & Principe tenu pour vrais & Éléments contextuels \\
\hline $\begin{array}{l}\text { Concevoir le cycle } \\
\text { et choisir les objets } \\
\text { d'apprentissage }\end{array}$ & $\begin{array}{l}\text { - Le cycle se conçoit à partir d'un } \\
\text { découpage de la compétence } \\
\text { attendue en objets d'enseignement } \\
\text { - Il faut faire travailler tous les } \\
\text { éléments constitutifs de la } \\
\text { compétence attendue }\end{array}$ & $\begin{array}{l}\text { Prescriptions } \\
\text { primaires : } \\
\text { programmes et fiches } \\
\text { ressources }\end{array}$ \\
\hline \multicolumn{3}{|c|}{ Responsabilité engagée } \\
\hline \multicolumn{3}{|c|}{$\begin{array}{l}\text { Permettre aux élèves de développer les compétences attendues définies par les } \\
\text { programmes }\end{array}$} \\
\hline
\end{tabular}

\section{Résultats}

Nous avons identifié deux grandes responsabilités qui selon le cas, sont engagées, souhaitées ou reportées par les stagiaires. La première renvoie à l'impératif de faire apprendre les élèves. La seconde renvoie à l'impératif d'éduquer les élèves.

Une telle distinction est sans doute discutable, nos résultats nous ont cependant conduit à procéder à ce découpage pour plus de clarté : nous distinguons ainsi les prescriptions dont l'objet est d'aider les enseignants à organiser leur travail en vue des apprentissages des élèves, de celles qui renvoient à des enjeux éducatifs plus généraux liés au bien-être et au devoir-être (Leleux, 2014). 


\section{eJRIEPS spécial 3 (janvier 2020)}

\subsection{Un impératif d'instruction}

4.1.1. Développer les compétences attendues

II s'agit en premier lieu pour les stagiaires de suivre les programmes et, plus précisément, de faire en sorte que les élèves puissent développer la compétence attendue définie par les programmes :

« J'ai découpé la compétence attendue, je me la suis bien relue, les fiches ressources surtout. Je me base sur ça au départ, parce que sinon on ne sait pas sur quoi partir. [...] Je découpe, je vois globalement les différents éléments et je me dis va falloir bosser tout ça. » (Tom, EAS)

«L'idée c'est de travailler tous les aspects de la compétence attendue et qu'ils puissent tout mobiliser ensemble 》 (Théo, EAS)

« J'avais la compétence, j'essayais de voir ce qui me semblait prioritaire, là où mes élèves semblaient avoir des difficultés. Maintenant, en général, tout est un petit peu lié donc voilà, et puis après donc voilà, j'ai essayé de trouver des situations qui correspondaient » (Marie, EAS)

\subsubsection{Permettre le réinvestissement des acquis}

II est impératif ensuite, pour l'ensemble des stagiaires, d'organiser les conditions d'enseignement de façon que les élèves puissent réinvestir leurs acquis dans des situations plus globales. Cette idée de réinvestissement les engage tout particulièrement :

« Le choix des couloirs... déjà je l'utilise parce qu'après on va sur du double et donc je voulais qu'ils réinvestissent ce qu'on allait voir » (Yan, EAS) « J'aime bien (les situations avec points bonus) parce que [...] ça donne un aménagement du milieu qui pour moi les oblige à mettre en place ce sur quoi on a travaillé avant » (Tom, EAS)

«Moi je sais que je ne fonctionne pas souvent sur des matchs ou c'est que des matchs [...] c'est quand même une opposition pour apprendre quelque chose ou bien pour remettre en place ce qu'on a essayé d'apprendre pendant la séance » (Tom, EAS)

Cette responsabilité est assumée à la fois lors de la préparation des séances et lors de la conduite des séances. Au moment de la préparation, les stagiaires s'efforcent de concevoir des situations d'apprentissage qui ont un lien entre elles (et ce dès l'échauffement) et qui s'enchaînent de façon logique au regard de l'objet d'apprentissage visé ou du thème de séance mis en avant. Ils prévoient alors ensuite une situation plus globale (par exemple : 


\section{eJRIEPS spécial 3 (janvier 2020)}

situations avec point bonus, matchs à thèmes) qu'ils proposent en fin de séance en vue de permettre le réinvestissement des acquis.

De la même façon, pendant la conduite de la séance (lors des phases d'explication collective - présentation et bilans intermédiaires - mais aussi lors des phases de régulation), ils veillent à focaliser l'attention des élèves sur les liens entre les différentes situations et leur intérêt pour les matchs :

«Pourquoi je vous ai fait travailler le service comme ça ? [...] C'est pour viser des zones précises qui mettent en difficulté l'adversaire. Donc en match, c'est ce qu'il faut chercher à faire. Là ça a pas trop marché mais en match gardez à l'esprit que en fonction du placement de l'adversaire si il est derrière, je vais plutôt jouer court et s'il est devant, je vais plutôt jouer long pour le mettre en difficulté. » (Théo, C).

II s'avère toutefois qu'une telle responsabilité est aussi parfois davantage souhaitée que réellement engagée. Tom l'exprime de la sorte :

«Mais du coup, là je me rends compte que je situe que par rapport à la tâche et que je les renvoie pas forcément sur les situations de jeu. Je leur dis : "Ça vous permet d'avoir le temps de faire ça et ça" et je le resitue pas, je le dis par rapport à l'exercice [...]. Je pense que mon intention c'était de faire comprendre que c'était utile dans le jeu mais du coup, je me rends compte que sur le moment je ne suis pas revenu dessus [...]. Je pense que c'est tellement logique dans ma tête que j'ai même pas pensé à l'expliquer. » (Tom, EAS)

4.1.3. Rendre explicite les savoirs appris et tisser des liens entre les séances Une troisième responsabilité, en lien avec la précédente, est celle de devoir rendre explicite les savoirs appris (le temps d'institutionnalisation) et de tisser des liens entre les séances. Cependant si la plupart des stagiaires sont convaincus qu'ils doivent assumer une telle responsabilité, ils sont en difficulté pour l'assumer pleinement :

« Je me force à le faire, je pense, parce que je pense que c'est vraiment important mais j'y pense pas forcément. Je me force à la faire parce que je me dis que c'est quand même bien de faire un petit retour à la fin, de poser les choses à plat et de dire : "Et bien voilà, on a appris ça quand même. Aujourd'hui on n'a pas fait que..., on n'a pas joué, on n'a pas fait que bouger nos bras, nos jambes, on a appris ça et la semaine prochaine on va apprendre ça ". » (Tom, EAS) 


\section{eJRIEPS spécial 3 (janvier 2020)}

" J'aime bien le faire (le bilan) pour leur amener un petit retour sur leur fonctionnement [...]. Et aussi, du coup, mettre un petit peu en perspective avec la prochaine leçon pour qu'il y ait un petit peu une cohérence dans les apprentissages proposés [...] mais c'est vrai qu'ils sont toujours assez pressés en fin d'heure, donc ça c'est compliqué. » (Théo, EAS)

"Ça permet à l'élève qui est vraiment à l'écoute de trouver du sens. J'ai tendance à faire ça, après ça dépend des fois, quand je sens que c'est nécessaire, je le fais sinon... » (José, EAC)

\subsubsection{Faire réfléchir les élèves sur leur pratique}

Une quatrième responsabilité évoquée par les stagiaires est celle d'avoir à organiser les conditions susceptibles d'amener les élèves à réfléchir sur leur pratique et à prendre conscience de ce qu'ils font et de ce qu'il faut faire pour réussir. L'extrait de l'entretien d'auto-confrontation croisée ci-dessous à propos d'une situation d'observation et de conseil mise en place par Théo en rend compte :

Théo : « Je pense que c'est vraiment primordial de se rendre compte que ce que l'on a travaillé en mettant les points bonus, [...] et qu'ils le voient sur le papier et qu'ils voient que quand ils le font pas, ils n'ont pas le même résultat. Sinon, si juste ils font le match, ils vont pas s'en rendre compte eux-mêmes. II faut les amener à réfléchir sur cette fiche [...]. »

Yan : «C'est une réflexion sur leur pratique ».

Cette responsabilité est là encore davantage souhaitée qu'engagée. Elle est même pour certains d'entre eux reportée. Faire réfléchir les élèves sur leur pratique passe en effet essentiellement pour les stagiaires dans la mise en place de situations au cours desquelles les élèves doivent s'observer, échanger ou se conseiller. Or, de telles mises en œuvre ne sont pas sans leur poser des problèmes et les questionner. Les échanges lors de l'entretien d'auto-confrontation croisée se poursuivent en effet de la façon suivante :

Tom : «Moi c'est vrai que je n'ai pas du tout mis en place de rôle d'observateur je trouve que c'est très très dur à mettre en place, parce que je pense que je suis débutant, pour l'instant je m'axe beaucoup sur le moteur [...], j'ai du mal à le mettre en place pour tout gérer. «

José : « Je pense que c'est pas forcément nécessaire de noter, je pense que tu peux leur faire apprendre l'amorti ou le dégagé sans qu'ils aient écrit ça sur un papier. 》 


\section{eJRIEPS spécial 3 (janvier 2020)}

Yan : " Je trouve intéressant qu'ils interviennent sur les adversaires, sur les partenaires [mais] je suis allé plus sur la facilité, ça demandait plus de travail de préparation de fiches, analyser les fiches. ”

José : «Le coaching [...] moi je trouve que c'est intéressant mais après sur le papier est-ce qu'ils sont capables de se coacher ? Est-ce que c'est vraiment utile ?»

Théo : "À mon avis, il faut vraiment les guider sur les conseils à donner, c'està-dire leur donner vraiment un critère, par exemple là, le service et les points à marquer, et juste les trois échanges qui suivent derrière. Faut juste qu'ils repèrent ça. »

\subsubsection{Conseiller et aider les élèves}

La dernière responsabilité assumée par l'ensemble des stagiaires est celle d'avoir à conseiller les élèves et de leur apporter les moyens de progresser. Les stagiaires s'efforcent tout particulièrement d'aller voir tous les élèves et, pour certains d'entre eux, plus particulièrement les élèves les plus en difficulté. Aider les élèves à progresser consiste essentiellement pour eux à leur dire ce qu'ils doivent faire ou bien à leur montrer comment réaliser tel ou tel geste :

"Ma mission c'est passer voir, passer la consigne et je regarde. [...] II faut que je vérifie » (Yan, EAC)

"Ce qu'on dit, [...] c'est en rapport avec la situation qui s'est passée précédemment. [...] et là on vient lui donner la solution, lui dire ce qu'il fallait faire. » (José, EAC)

« J'essaie surtout d'expliquer, oui de leur expliquer comment bien faire, j'essaie de trouver des astuces pour que ça leur parle, pour qu'ils le fassent. » (Marie, EAS)

"(Je montre) pour qu'ils comprennent bien ce que j'attends, pour que je sois sûre qu'ils soient dans ce que j'attends, je montre ce que je veux moi. » (Marie, EAS)

\subsection{Un impératif d'éducation}

4.2.1. Favoriser le bien-être des élèves : un impératif d'épanouissement La première responsabilité engagée par les stagiaires renvoie à un impératif d'épanouissement des élèves. Elle consiste à devoir mettre en confiance les élèves, à les valoriser et à faire en sorte qu'ils se sentent reconnus. Pour ce faire, les stagiaires 


\section{eJRIEPS spécial 3 (janvier 2020)}

s'efforcent d'être bienveillants, d'encourager les élèves, de montrer leur présence et leur attention, de mettre en avant les réussites et de les féliciter :

"Aller voir chaque élève et donner au moins un petit feed-back à chacun histoire [...] qu'il sache qu'on est derrière, qu'on voit ce qu'il fait, qu'on lui donne des conseils, qu'on s'occupe de chacun. " (Tom, EAC)

" J'essaye d'être un maximum sur les encouragements, les feed-back positifs. Le feed-back négatif je l'utilise très très rarement. » (Yan, EAS) "L'idée c'est voilà de, je suis content d'eux. Donc j'essaie de leur dire. Je suis tombé sur un coup, il a bien joué donc je l'ai félicité, voilà. [...] Parce que c'est valorisant pour un enfant de sentir que, qu'il est reconnu par un adulte. " (José, EAS)

"Il essaie de rassurer les élèves : "C'est bien ce que tu fais, c'est bien d'essayer de faire ça, c'est pas grave si tu as fait ça. » (Tom, EAC).

La seconde responsabilité tient à l'obligation de créer un climat de classe agréable, de procurer du plaisir aux élèves et de faire en sorte qu'ils s'amusent. II est important par conséquent pour eux de choisir avant tout des situations ludiques qui procurent du plaisir et de l'amusement:

"C'est ludique. Je suis sûr qu'ils vont se mettre dedans, qu'ils vont rigoler, qu'ils vont accrocher au truc. Et je trouve, on parle de compétence transversale à acquérir, je pense que le plaisir de pratiquer, de venir, de s'amuser, de s'impliquer, c'est quelque chose de transversal à installer. " (Yan, EAC)

" J'aime bien le fait que ce soit une situation ludique, moi j'essaie dans mes échauffements de faire [...] un petit truc qui peut les faire un peu rigoler. » (José, EAC)

"Ils s'amusent, à partir du moment où ils s'amusent. » (Yan, EAC)

Cette quête du plaisir chez les élèves est à ce point importante que certains d'entre eux tiennent à s'en assurer auprès des élèves :

"À la fin de chaque séance, j'essaye souvent de dire : "Est-ce que vous avez pris du plaisir dans la séance ? "Parce que pour moi, c'est quelque chose d'important qu'ils en prennent, parce que l'activité physique en elle-même elle procure du plaisir, et en plus je pense que ça aide aux apprentissages. Et si jamais il y a un bon climat de classe et qu'ils s'amusent, ce sera plus facile pour apprendre des choses. » (Tom, EAC) 


\section{eJRIEPS spécial 3 (janvier 2020)}

4.2.2. Apprendre aux élèves à vivre ensemble : un impératif de socialisation La dernière responsabilité renvoie à un impératif de socialisation. Celle-ci cependant n'a été évoquée que par un seul des 5 stagiaires. Pour lui, il est de sa responsabilité d'avoir à favoriser les relations sociales, de faire en sorte que les élèves échangent et se sentent partie prenante d'un groupe ou d'une équipe :

«Mon projet, c'est le groupe... Pour moi l'éducation physique et sportive, il faut que ce soit socialisant. C'est ma vision des choses. [...] C'est ma vision de l'éducation physique et sportive, et pas que de l'éducation physique et sportive d'ailleurs, de l'éducation en général. ”

"Ça aussi, limite ça m'embête de devoir le rappeler à chaque fois : "Quand vous avez terminé, vous allez encourager vos partenaires [...] Ça ça n'allait pas dans l'esprit que je voulais leur inculquer, on est une équipe, on fait partie d'une équipe même quand on ne joue pas. " (Yohan, EAS)

Pour les autres en revanche, cet impératif de socialisation, bien qu'étant un enjeu éducatif social majeur repris à travers l'expression notamment des finalités de l'EPS, n'est pas une obligation morale (Jonas, 1979).

\section{Discussion}

\subsection{Entre mission d'instruction et d'éducation : des responsabilités difficiles à assumer}

Ces résultats montrent que les stagiaires observés se sentent responsables à la fois de l'instruction des élèves et de leur éducation. La confrontation au " choc de la réalité » ne leur fait pas perdre de vue leur mission de transmission des savoirs. Pour autant, nombreuses sont les responsabilités qu'ils disent ne pas assumer véritablement. Ainsi, sontelles pour certaines d'entre elles seulement souhaitées (rendre explicites les savoirs appris et tisser des liens), voire même parfois reportées (faire réfléchir les élèves sur leur pratique) ou ignorées (développer la socialisation). De fait, si les stagiaires font partie de ceux qui ne renoncent pas aux idéaux pédagogiques promus dans le cadre de la formation (Daguzon \& Goigoux, 2012), ils redéfinissent toutefois les prescriptions lors du travail réel en classe afin de préserver au mieux leur zone de confort (Ria, 2017). Comme le soulignent Saujat \& Serres (2015, p. 15) «ce n'est que lorsqu'ils auront atteint un premier palier d'efficacité conditionné par un usage de soi générant confort et plaisir professionnels », qu'ils s'interrogeront sur les modalités selon lesquelles ils peuvent eux-mêmes constituer des moyens plus efficaces au service de l'activité d'apprentissage des élèves. 


\subsection{Instruire plutôt qu'éduquer}

II s'avère ensuite que les prescriptions que les stagiaires s'auto-prescrivent renvoient davantage aux prescriptions primaires et secondaires liées aux apprentissages des élèves qu'aux enjeux éducatifs, tout au moins à ceux relatifs à la question de la socialisation ou du « vivre ensemble » (MENESR, 2015b). Ainsi les stagiaires observés veulent, en y parvenant plus ou moins bien : concevoir des situations susceptibles de développer les compétences attendues ; conseiller et aider les élèves ; tisser des liens entre les séances et expliciter les acquis ; conduire les élèves à identifier les procédures efficaces en les faisant réfléchir sur leur pratique ; et enfin, leur apprendre à mobiliser leurs acquis dans des situations de réinvestissement. Ces intentionnalités ou «vouloir-faire » (Saujat \& Serres, 2015) ont une certaine proximité avec les gestes professionnels préconisés en formation qui actualisent cinq préoccupations enchâssées mises en évidence par Bucheton et Soulé (2009). Nos résultats rejoignent de ce point de vue ceux que d'autres auteurs ont pu mettre en évidence : les stagiaires sont particulièrement sensibles aux recommandations ou prescriptions primaires qui proviennent de l'institution scolaire, mais aussi et surtout aux prescriptions secondaires qui émanent des instituts de formation professionnelle (Goigoux, 2007). Nous pouvons considérer que ces prescriptions fonctionnent chez les stagiaires « comme une mémoire agissante consubstantielle de l'activité » (Amigues, 2009, p. 16) même si, comme l'a montré Daguzon (2009), ces derniers rendent parfois les prescriptions moins exigeantes, notamment en reportant à plus tard les moments au cours desquels les élèves sont placés en situation d'observation, de coaching ou d'analyse collective de leurs actions.

\subsection{Le plaisir des élèves en EPS : épanouissement ou amusement ?}

Si les prescriptions primaires et secondaires engagent tout particulièrement la responsabilité d'instruction des stagiaires, il apparaît que les stagiaires se sentent également « obligés » par le bien-être des élèves, et plus précisément par leur plaisir. Ainsi, alors que Daguzon et Goigoux (2007) relèvent chez les jeunes enseignants une grande homogénéité des conceptions selon lesquelles les élèves doivent être actifs, motivés et prendre la parole, les stagiaires observés semblent considérer que les élèves doivent avant tout prendre du plaisir et s'amuser. Nos résultats rejoignent dès lors ceux mis en évidence par ailleurs : les stagiaires se souciant en priorité de mettre les élèves en activité recourent à des activités ludiques (Férone, 2010) ; agir de la sorte leur permet de susciter et de maintenir l'engagement des élèves dans les tâches proposées (Goigoux et al, 2009). II 


\section{eJRIEPS spécial 3 (janvier 2020)}

convient toutefois de nuancer de tels propos. Si les stagiaires accordent une telle importance au plaisir des élèves en EPS, c'est aussi sans doute du fait de leur rapport positif à la pratique des APSA. Tom l'exprime de la façon suivante : "l'activité physique en ellemême elle procure du plaisir ». De fait, ne pas éprouver de plaisir c'est en quelque sorte, pour les stagiaires, passer à côté de ce qui constitue «la nature » même des activités physiques, et par conséquent de l'EPS. Aussi, ces derniers considèrent-ils comme une évidence le fait que les élèves doivent prendre du plaisir et s'amuser en EPS : " Ils s'amusent! À partir du moment où ils s'amusent...! " (Yan). II s'avère cependant qu'amusement et plaisir sont radicalement différents (Lavie \& Gagnaire, 2014). Ainsi, alors que pour Lavie et Gagnaire (2014) le plaisir en EPS, qui ne va jamais de soi pour les élèves, est une finalité éducative, en ce sens qu'il favorise l'épanouissement de la personne, et donc sa puissance d'exister, pour les stagiaires il est probablement davantage un objectif à court terme. Ce «vouloir-faire » renverrait par conséquent davantage à des prescriptions atténuées ou réduites à leur plus simple expression : favoriser le bien-être et (r)éveiller le plaisir d'agir des élèves en EPS (Lavie \& Gagnaire, 2004) consistant ici à proposer des situations ludiques susceptibles d'amuser les élèves.

\subsection{Le réinvestissement des acquis en EPS : routine professionnelle ou} démarche constructive ?

Le dernier point qui ressort de nos résultats renvoie à la volonté de favoriser le transfert ou le réinvestissement des apprentissages en EPS. Il questionne l'utilité de certaines pratiques sur les apprentissages des élèves. II apparaît en effet que les stagiaires observés reproduisent systématiquement la routine professionnelle dénoncée par Falco (2013) : des situations d'apprentissages plus ou moins analytiques qui se succèdent conduisant vers une situation plus globale en fin de séance dans laquelle les élèves doivent réinvestir ce qu'ils ont vu auparavant. Si les stagiaires agissent de la sorte, c'est en premier lieu parce qu'ils n'envisagent pas d'aller à l'encontre des manières habituelles d'agir des enseignants d'EPS envers leurs élèves. Ils répondent de ce point de vue aux prescriptions ascendantes qui remontent des collectifs de travail (Saujat \& Serres, 2015) : « ils (les élèves) ont [...] l'habitude, tous les ans ils font ça, c'est toujours des situations puis ça finit par un match. Ils le savent. " (Théo, EAC). Mais c'est aussi vraisemblablement parce que le transfert qui se situe au cœur de l'acte d'enseignement-apprentissage (Bissonnette et Richard, 2001), est considéré comme un allant de soi : " Je pense que c'est tellement logique dans ma tête (le lien entre l'exercice proposé et le jeu qui suit) que j'ai même pas pensé à l'expliquer » (Tom). 


\section{eJRIEPS spécial 3 (janvier 2020)}

Les stagiaires peuvent alors avoir tendance à mobiliser « ce qu'il est d'usage de faire " sans se préoccuper de savoir si les conditions d'apprentissage sont effectivement favorables au réinvestissement des acquis. II faudrait en effet déjà pour cela qu'ils puissent identifier et objectiver les acquis réalisés. Or, rien n'est moins sûr. De fait, bien que sensibles à l'idée de gestes de tissage nécessaires aux transferts d'apprentissage (Bucheton \& Soulé, 2009), ils reproduisent, sans chercher à l'interroger, un schéma d'intervention pré-établi a priori discutable (Falco, 2013), sorte de vulgate pédagogique (Rayou, 2014) résultant de la question délicate et controversée du transfert des apprentissages moteurs et non moteurs.

\section{Conclusion : implications pour la formation}

Ces quelques résultats, portant sur un nombre réduit de stagiaires, sont à enrichir par des recherches de plus grande envergure. Nous avons toutefois tenté de montrer qu'ils pouvaient aider à extraire une argumentation de portée plus générale (Passeron \& Revel, 2005). Rendant compte du "vouloir-faire» ou de ce dont se sentent responsables des stagiaires en EPS qui entrent dans le métier, et ainsi de ce qui les engage, ils apportent en outre aux formateurs des points d'ancrage utiles pour repérer les objets à travailler en formation. II en va ainsi notamment des questions relatives au plaisir des élèves, aux conditions d'apprentissage favorables au réinvestissement, ou à la réflexion sur la pratique. Mais ce sont aussi les questions liées au développement du vivre ensemble qui gagneraient à être abordées.

Plus généralement, de tels résultats invitent à concevoir des modalités de formation susceptibles de conduire les stagiaires à prendre conscience de ce qui les engage, de ce qu'ils négligent ou reportent au regard des éléments contextuels qui organisent leur activité ; prendre conscience par exemple de ce qu'ils font par adhésion à des doxas, des vulgates pédagogiques ou des succédanés de préconisations (Rayou, 2014). Nous rejoignons dès lors Rayou (2018) qui suggère, pour éviter « l'assoupissement » des enseignants dans des doxas, de mettre ces derniers dans des situations de controverse professionnelle (Saujat, 2010).

Les entretiens d'autoconfrontation croisée, conduits cette fois-ci dans une perspective de développement professionnel (Duboscq \& Clot, 2010), paraissent particulièrement intéressants à cet égard. La démarche, selon cette perspective, consiste à provoquer et à élargir le dialogue entre les sujets et en eux, en vue du développement de leur activité concrète. L'autoconfrontation peut ainsi devenir un instrument de transformation de l'activité. Des «mouvements» transformatifs apparaissent d'ailleurs dans notre proche 


\section{eJRIEPS spécial 3 (janvier 2020)}

recherche. Ainsi, peut-on identifier des moments de «mise au travail » du collectif de stagiaires au cours desquels apparaissent des dilemmes et des conflits dans l'activité dialogique sur la question des responsabilités ou du "vouloir faire " (faire réfléchir les élèves par exemple), susceptibles de provoquer des "mouvements" conduisant les stagiaires à se développer. Ce sont de tels mouvements qu'il nous paraît important de provoquer dans des dispositifs conçus à cette fin.

\section{Bibliographie}

Amigues, R. (2009). Le travail enseignant : prescriptions et dimensions collectives de l'activité. Les Sciences de l'éducation pour l'Ëre nouvelle, 42(2), 11-24.

Bissonnette, S., \& Richard, M. (2001). Comment construire des compétences en classe. Des outils pour la réforme. Montréal : Chenelière/McGraw-Hill.

Bucheton, D., \& Soulé, Y. (2009). Les gestes professionnels et le jeu des postures de l'enseignant dans la classe : un multi-agenda de préoccupations enchâssées. Éducation \& didactique, 3(3), 29-48.

Butlen, D., Charles-Pézard, M., \& Masselot, P. (2009). Gestes et routines professionnels : un enjeu pour analyser et intervenir sur les pratiques enseignantes. Actes Colloque international Espace Mathématique Francophone, Dakar, 6-10 avril 2009.

Clot, Y., Prot, B., \& Werthe, C. (2001). Clinique de l'activité et pouvoir d'agir. Éducation permanente, 146, $12-37$.

Daguzon, M. (2009). De la prescription à la redéfinition de la tâche d'enseignement (étude longitudinale). In R. Goigoux, L. Ria, et M. C. Toczek-Capelle (Dir.), Les parcours de formation des enseignants débutants (pp. 101-120). Clermont-Ferrand : Presses Universitaires Blaise Pascal.

Daguzon, M., \& Goigoux, R. (2007). L'influence de la prescription adressée aux professeurs des écoles en formation initiale : construction d'un idéal pédagogique. CDRom Actes du colloque AREF : Strasbourg. http://www.congresintaref.org/actes_site.php

Daguzon, M., \& Goigoux, R. (2012). Apprendre à faire classe. Les apprentissages professionnels des professeurs des écoles en formation par alternance. Revue française de pédagogie, 181, 27-42.

Daniellou, F. (2002). Le travail des prescriptions. Conférence inaugurale au 37ème Congrès de la SELF, les évolutions de la prescription, Aix-en-Provence. http://www.ergonomie-self.org/actes/congres2002.html 


\section{eJRIEPS spécial 3 (janvier 2020)}

Deguilhem, P., \& Juanico, R. (2016). Promouvoir l'activité physique et sportive pour tous et tout au long de la vie: Des enjeux partagés dans et hors de l'école. Rapport remis au premier ministre Manel Valls.

Duboscq, J., \& Clot, Y. (2010). L'autoconfrontation croisée comme instrument d'action au travers du dialogue : objets, adresses et gestes renouvelés, Revue d'anthropologie des connaissances, 2(4), 255-286.

Falco, A. (2013). Situations complexes en EPS : la nécessité d'accompagner la conception et les mises en œuvre d'un enseignement par compétences. Enseigner l'EPS, Revue de l'AE-EPS, 261, 21-28.

Ferone, G. (2010). Les conceptions du métier chez des professeurs des écoles en formation. In Congrès international d'actualité de la recherche en éducation et en formation (AREF, Genève). En ligne : http://www.unige.ch/aref2010/index.html

Lavie, F., \& Gagnaire, P. (2014). Plaisir et processus éducatif en EPS. Saint-Mandé : Edition AE-EPS.

Goigoux, R. (2007). Un modèle d'analyse de l'activité des enseignants. Éducation \& Didactique, 3(1), 19-41.

Goigoux, R., Ria, L., \& Toczek-Capelle, M. C. (2009). Les parcours de formation des enseignants débutants. Clermont-Ferrand : Presses Universitaires Blaise Pascal.

Jonas, H. (1979). Le principe responsabilité, trad. française. Paris : Les Éditions du Cerf.

Lavie, F., \& Gagnaire, P. (2014). Plaisir et processus éducatif en EPS. Saint-Mandé : Edition AE-EPS.

Leplat, J. (1997). Regards sur l'activité en situation de travail. Paris : PUF.

Leleux, C. (2014). Instruire et éduquer sur fond d'éthique. Pratiques [En ligne], 163-164, mis en ligne le 31 décembre 2014, consulté le 30 avril 2019. URL : http://journals.openedition.org/pratiques/2237 ; DOI : 10.4000/pratiques.2237

Magendie, E. (2014). Organisateurs de l'activité et rapport au curriculum recommandé des étudiants-stagiaires d'EPS en formation par alternance. Questions Vives. Recherches en éducation, 22, 83-104.

Magendie, E. (2016). Développement de la conceptualisation dans l'activité de conception d'étudiants-stagiaires en EPS durant l'année de formation en alternance. Recherches en éducation, Hors-série 9, 58-72

Mayen, P., Ferron, O., Humblot, J.-P., \& Bazile, J. (2006). Introduire un référentiel de situations dans les référentiels de diplôme en BTS. Rapport de recherche de l'unité propre «Développement professionnel et formation » pour le ministère de 
eJRIEPS spécial 3 (janvier 2020)

l'Agriculture. Département des Sciences de la formation et de la communication, Enesad.

MENESR (2015a). Arrêté du 9-11-2015 - J.O. du 24-11-2015.

MENESR (2015b). Décret n²015-372 du 31-3-2015 - J.O. du 2-4-2015

Miles, M. B., \& Huberman, A. M. (2003). Analyse des données qualitatives. Bruxelles : De Boeck Université.

Paillé, P., \& Mucchielli, A. (2003). L'analyse qualitative en sciences humaines et sociales. Paris : Armand Colin.

Passeron, J.-C., \& Revel, J. (Eds.) (2005). Penser par cas. Paris : Éditions de l'École des Hautes Études en Sciences Sociales.

Pastré, P. (2011). La didactique professionnelle : approche anthropologique du développement chez les adultes. Paris : Presses universitaires de France.

Pastré, P., Mayen, P. \& Vergnaud, G. (2006). La didactique professionnelle. Revue française de pédagogie, 154, 145-198.

Rayou, P. (2008). Ni guerre, ni paix. Tensions et malentendus dans la formation. In Perrenoud, P., Altet, M., Lessard, C., et Paquay, L. (Eds.) Conflits de savoirs en formation des enseignants (pp. 77-89). Bruxelles : De Boeck.

Rayou, P. (2014). Prescriptions et réalités du travail enseignant, Impasses et ouvertures de l'accompagnement en formation. In Paquay L., Perrenoud, P., Altet, M., Etienne, R., et Desjardins, J. (2014). (Eds.) Travail réel des enseignants et formation (pp. 35-47). Bruxelles : De Boeck.

Rayou, P. (2018). Conclusion: Savoirs et conceptions professionnelles des enseignants. Éducation \& Formation, e-310, 97-100.

Ria, L. (2017). Premiers pas professionnels : trois composantes typiques de l'activité novice des enseignants. In J.M Barbier, et M. Durand (Eds.), Encyclopédie d'analyse des activités (pp. 437-458). Paris : PUF.

Roux-Perez, T. (2012). Construire la professionnalité enseignante à l'heure des réformes : enjeux de - et défis pour - la formation. In T. Pérez-Roux (Ed.), La professionnalité enseignante (pp. 97-120). Rennes : Presses Universitaires de Rennes.

Saujat, F. (2004). Spécificités de l'activité d'enseignants débutants et "genres de l'activité professorale". Polifonia, 8, 67-94.

Saujat, F. (2010). Travail, formation et développement des professionnels de l'éducation : voies de recherche en sciences de l'éducation. Note de synthèse pour l'Habilitation à diriger des recherches. Université de Provence, Aix-Marseille. 


\section{eJRIEPS spécial 3 (janvier 2020)}

Saujat, F., \& Serres, G. (2015). L'activité de l'enseignant d'EPS entre préoccupations et «occupations » : un point de vue développemental. eJRIEPS, 34, 4-30.

Saussez, F. (2014). Une entrée activité dans la conception d'environnements de formation pour sortir d'une vision fonctionnaliste de la formation, un essai de conclusion. Activités, 11(2), 188-200. http://www.activites.org/v11n2/v11n2.pdf

Tourmen, C. (2014). Usages de la didactique professionnelle en formation : principes et évolutions. Savoirs, 36, 9-40.

Vergnaud, G. (1996). Au fond de l'action, la conceptualisation. In J.-M. Barbier (Ed.), Savoirs théoriques et savoirs d'action (pp. 275-292). Paris : PUF.

Yvon, F., \& Garon, R. (2006). Une forme d'analyse du travail pour développer et connaître le travail enseignant : l'auto-confrontation croisée. Recherches qualitatives, 26(1), 51-80. 